\title{
Session 4:
}

\section{Atomic AND Molecular Gas IN GALAXIES:}

Cooling Flows, Radio Galaxies,

High-redshift Galaxies, Epoch of Reionisation 


\title{
Brightest Cluster Galaxies \& Cooling Flows
}

\author{
Philippe Salomé \\ LERMA, Observatoire de Paris \\ 61 av. de l'Observatoire, F-75014 Paris, France \\ email: philippe.salome@obspm.fr
}

\begin{abstract}
In the absence of any form of feedback heating, the gas in the central regions of massive relaxed cluster should cool and initiate a cooling flow. The presence/efficiency of an additional heating and the ultimate fate of the cooling gas is the subject of an extensive debate. In the last decade, molecular and atomic gas have been found in many Brightest Cluster Galaxies. I will review these observational results and discuss their implication for galaxy formation/evolution, in the perspective of ALMA.
\end{abstract}

Keywords. galaxies: cooling flows - intergalactic medium - galaxies: elliptical and lenticular, $\mathrm{cD}$

\section{The Brightest Cluster Galaxies and the Intra-cluster Medium}

Brightest Cluster galaxies (BCGs) are giant elliptical galaxies standing in the centre of galaxy cluster potential wells. Most of the mass of galaxy clusters lies in the Intracluster Medium (ICM), a very hot plasma that emits in the X-ray. A large fraction of galaxy clusters contains a cooling flow (Fabian et al., 1994). BCGs are thus interesting nearby sources to study intergalactic gas cooling/accretion onto galaxies; they are also good laboratories to study the effect of AGN-feedback that may prevent the hot gas from cooling and eventually form stars; two important mechanisms of galaxy formation.

Cool Core Clusters in the X-ray. Cool core clusters (also called cooling flows) are found in about $50-66 \%$ of the galaxy clusters identified in the X-ray. The last generation of Xray satellites, Chandra and XMM-Newton, with respectively a higher spatial and spectral resolution, showed that inside the central $200 \mathrm{kpc}$, the hot gas has enough time to cool; the hot gas cooling time being as short as a few $100 \mathrm{Myr}$ in the central $50 \mathrm{kpc}$ (Allen et Fabian, 2001; Voigt et al. 2003). However the strengths of cooling flows, measured by the so-called X-ray mass deposition rates, were corrected by about one order of magnitude when compared to the numbers derived from ROSAT and the previous generation of X-ray satellites (Peterson et al., 2001), leading to $\mathrm{dM}_{\mathrm{X}} / \mathrm{dt} \sim 10-100 \mathrm{M}_{\odot} / \mathrm{yr}$.

$A G N$-feedback to regulate cooling flows. A large amount of work has been done in the last decade to study the different feedback processes that could prevent the hot gas from cooling and reduce the mass deposition rates (conduction, SN-feedback, AGN-feedback...). Comparisons of high resolution X-ray images of cluster centres with the radio-emission of the central AGN showed the existence of large X-ray cavities (also called bubbles) that are filled by the radio-jet emission ejected from the central engine. This was the first clear evidence of an interaction between the AGN and the ICM in favor of an energetic feedback coming from the AGN (see Fabian et al., 2012 for a review).

\section{Molecular and atomic gas in cooling flows}

Before the last decade, BGCs were not considered as possible reservoir of molecular gas. Many attempts had be performed with single dish telescopes and interferometers, 


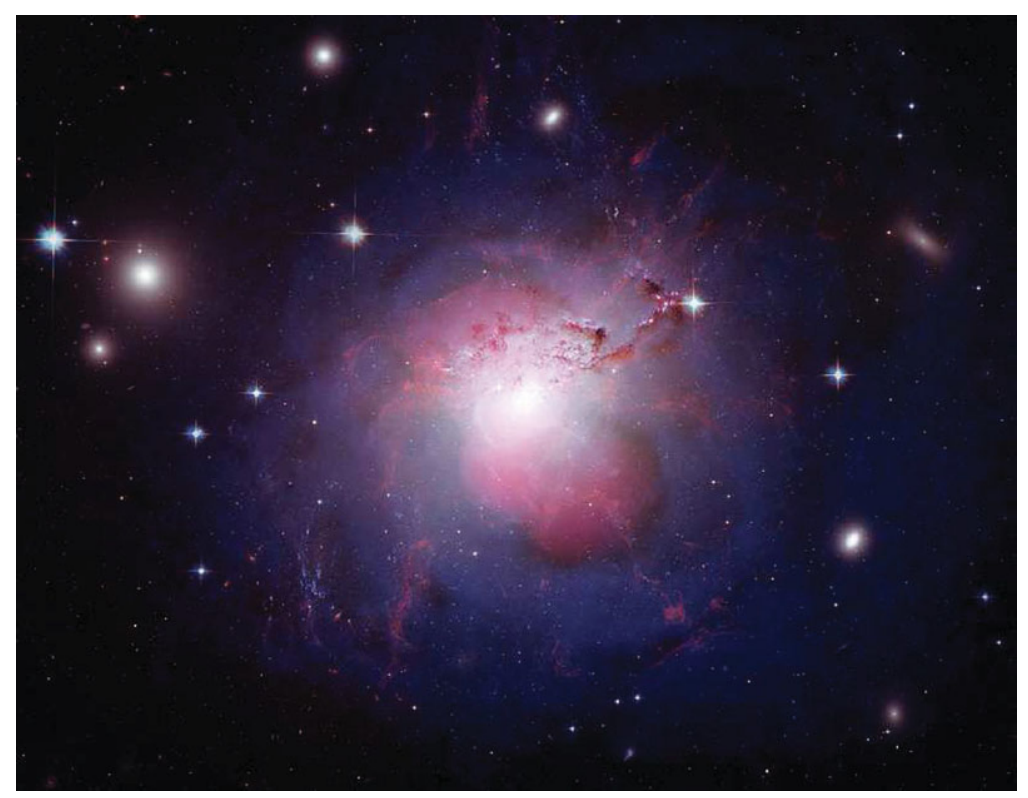

Figure 1. Radio-lobes at $328 \mathrm{MHz}$ in pink. X-ray emission $[0.3-7 \mathrm{keV}]$ in violet: diffuse ICM emission and shells around the lobes. Optical data in red, green and blue (stars, filaments, background galaxies, dust-lane; see the on-line proceedings for a color version of this figure). Credit: NASA CXC/IoA/A.Fabian et al.; NRAO/VLA/G. Taylor; NASA/ESA/Hubble Heritage (STScI/AURA) \& Univ. of Cambridge/IoA/A. Fabian.

to detect a cold gas reservoir built up from the ICM cooling (Grabelski et Ulmer, 1990, McNamara et al. 1994, O’Dea et al., 1994, Braine et Dupraz 1994). But no trace of any molecular gas emission was found apart in NGC 1275 that was the only source detected in CO emission lines (Lazareff et al., 1989; Mirabel, 1989; Reuter et al., 1993; Braine et al., 1995; Inoue et al., 1996; Bridges et al., 1998). But it was suspected to be a merger. Cold gas in a hot environment: the first CO detections Molecular gas has now been detected mostly via $\mathrm{CO}(1-0)$ and $\mathrm{CO}(2-1)$ in about 30 BCGs (Edge et al., 2001; Salomé \& Combes, 2003) with the IRAM 30m-telescope and the JCMT. The mass of molecular gas in these galaxies is $\sim 10^{8-11.5} \mathrm{M}_{\odot}$. So large amounts of gas exist in these giant elliptical galaxies and represent about $10 \%$ of what is expected if it was cooling from the hot ICM at rates derived from the X-ray observations. These surveys also showed that the amount of molecular gas correlates with the X-ray mass deposition rates and with the $\mathrm{H}_{\alpha}$ luminosities (most of systems with $\mathrm{L}\left(\mathrm{H}_{\alpha}\right)>10^{41} \mathrm{erg} \mathrm{s}^{-1}$ being also detected in CO emission lines). No clear anticorrelation was found with the AGN radio emission.

Other molecules and atomic lines. Dense gas or shocked gas tracers (i.e. CN, HCN, HCO+, $\mathrm{SiO})$, were observed but are below the detection limit of current mm-facilities. Only NGC 1275 was detected in $\mathrm{HCN}(3-2)$ by Salomé et al., (2008), CN(2-1), $\mathrm{C}_{2} \mathrm{H}(3-2)$ and $\mathrm{HCO}+(3-2)$ by Bayet et al., (2011). Comparisons with Photodissociation Region (PDR), X-ray Dominated region (XDR), Cosmic-Rays dominated Regions (CRDR) modeling start to be possible (Bayet et al., 2010) and ALMA will open a new era for understanding the physical conditions of the molecular gas in BCGs. Herschel detected FIR atomic lines (CII, OI, NII) and continuum dust emission. The derived line diagnostics are like typical star forming galaxies (Edge et al., 2010a,b). Large scale mapping of Perseus and Centaurus were even possible and showed different line excitation conditions for those two objects (Mittal et al., 2011, 2012). In the case of Centaurus, in order to explain the 
atomic and optical spectra, another source of heating than the one provided by PDR and HII regions is required (i.e. cosmic rays, shocks or reconnection diffusion).

Evidence of a multiphase ICM and star formation in BCGs In the last decade, tracers of gas at different temperatures were also detected: in the X-ray (Fabian 1994), the UV via the OVI line (Bregman et al., 2001), the optical via the $\mathrm{H}_{\alpha}$ emission (Heckman et al., 1989, Crawford et al., 1999, Mc Donalds et al., 2011 and references therein), the near-IR and mid-IR, via the ro-vibrational (Donahue et al., 2000, Edge et al., 2002) and the pure rotational $\mathrm{H}_{2}$ lines (Egami, 2006, Johnstone et al., 2007, Donahue et al., 2011). BCGs are also places of star formation. Star formation rates estimated from the the mid-IR (O'Dea et al., 2008), the FUV (O'Dea et al., 2010) and the FIR (Edge et al., 2010) are consistent and of the order of $1-50 \mathrm{M}_{\odot} / \mathrm{yr}$, typical of local star forming galaxies and about one order below the X-ray mass deposition rates. The molecular gas found in BCGs is thus a reservoir of material available to fuel the star formation. But what triggers the star formation in BCGs is still an open question.

\section{Where is the cold gas?}

Single dish telescopes and current mm-submm receivers have shown the presence of large amount of molecular gas in BCGs. Interferometers then looked at the spatial distribution and kinematics of the $\mathrm{CO}$ emission in the strongest systems, aiming at finding a possible signature of a cooling flow origin. Edge et Frayer (2003) showed with OVRO that most of the emission seems to be concentrated within the central $<20 \mathrm{kpc}$ around the central galaxy in Abell 1068, Zw3146, RXJ0821+07, A1835, RXJ 0338+09. Observations of NGC 1275 seem however to show that the emission could be more extended $\sim 50 \mathrm{kpc}$ (Salomé et al., 2011). At the distance of NGC $1275,1^{\prime \prime}$ is $370 \mathrm{pc}$, so this BCG is not only the strongest $\mathrm{CO}$ emitter of its kind, but it also a perfect target for sensitive and relatively high spatial resolution observations both at small scales around the AGN at very large distances from the centre.

At small scales: evidence for AGN fueling Observations of the central 40" (14kpc) of NGC 1275, with the Heterodyne Receiver Array (HERA) on the IRAM 30m-telescope, showed that the $\mathrm{CO}(2-1)$ emission was extended along the East-West axis, following the $\mathrm{H} \alpha$ emission (Salomé et al., 2006). A velocity map of this region showed no sign of rotation, but positive velocities on both sides of the centre and negative velocities close to the AGN. This can be the sign of infalling or outflowing gas. Lim et al. (2008) and Ho et al., (2009) confirmed this trend with the Submillimeter Array (SMA) and proposed a model of free falling gas to explain the velocity field inside the same region. Recent single dish observations (Salomé et al., 2011) pointed out a double horn profile in the central 11" (4kpc). Even closer to the AGN, Wilman et al., (2005) showed evidence of rotation inside the $1^{\prime \prime}(370 \mathrm{pc})$, with long slit observations of the $\mathrm{H}_{2}$ ro-vibrational line. All those observations point towards multiscale evidences of gas accretion onto the AGN. In Abell 1795, the only other source where the CO is resolved in kinematically separated components, Salomé et al., (2004) reported a molecular gas velocity gradient $(\sim 300 \mathrm{~km} / \mathrm{s}$ over $\sim 6 \mathrm{kpc})$ that can also be explained by gas accretion onto the AGN. The CO morphology, in this source, follows the one seen in X-ray, optical and UV: (i) along the northern radio-lobe and (ii) around the AGN.

At large scales: a very extended network of molecular filaments NGC 1275, in the centre of the Perseus cluster is famous for its huge filamentary structure seen in the optical (Conselice et al., 2001 and references therein) and in soft X-ray (Fabian et al., 2006). These filaments were detected in $\mathrm{H}_{2}$ by Hatch et al. (2005). The IRAM-30m telescope and the Plateau de Bure interferometer showed that these filaments are made of 


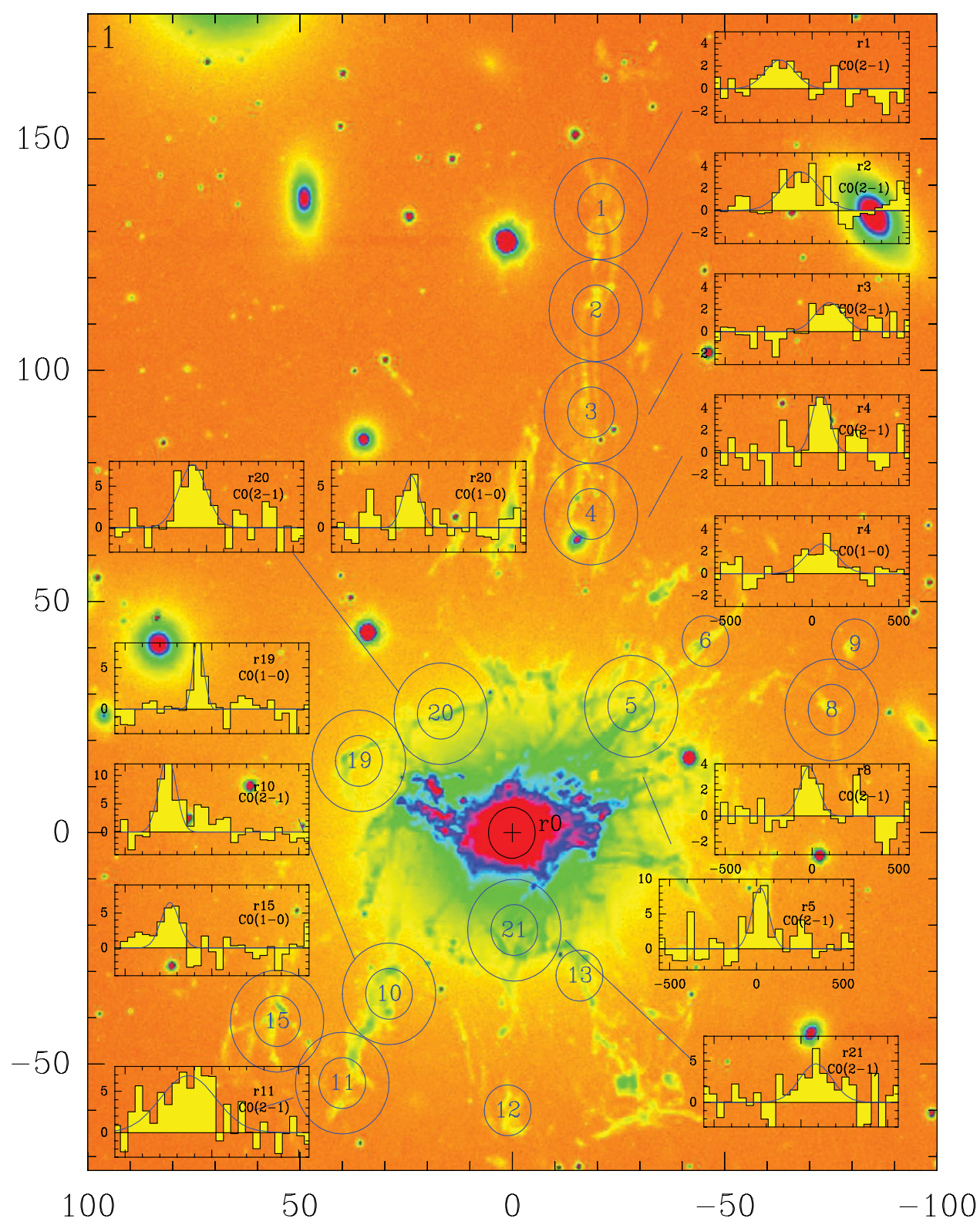

Figure 2. CO spectra superposed on the $\mathrm{H} \alpha$ filament structure around NGC 1275 (Conselice et al., 2001). The double circles show the 1.3 and $3 \mathrm{~mm}$ beamwidths at the regions where CO was detected. $\mathrm{CO}(1-0)$ and $\mathrm{CO}(2-1)$ spectra are shown when detected. The channel width is 42 $\mathrm{km} / \mathrm{s}$ and the $\mathrm{y}$-axis is main beam brightness temperature, in $\mathrm{mK}$. The velocity scale is from -560 to $560 \mathrm{~km} / \mathrm{s}$ in each insert. Small circles $(1.3 \mathrm{~mm}$ beamwidth) show regions where no CO was detected. The image size is $200^{\prime \prime} \times 250^{\prime \prime}$ ie $74 \mathrm{kpc} \times 92.5 \mathrm{kpc}$ (Salomé et al., 2011).

molecular gas, with the bulk of the mass lying in the form of very cold gas (Salomé et al., 2006; 2008a,b, 2011). The $\mathrm{CO}(2-1)$ emission line is detected at distances as far as $\sim 50 \mathrm{kpc}$ from the central galaxy in a thin and elongated filament, the outermost region containing up to $\sim 10^{7} \mathrm{M}_{\odot}$ of cold gas. This very distant filament has not only the same morphology, but also the same dynamics in $\mathrm{CO}$ and $\mathrm{H}_{\alpha}$, over a distance of $\sim 35 \mathrm{kpc}$. 
Evidences for AGN-driven cold filaments It is difficult to explain the presence of molecular gas at such large distances from the galaxy. How could molecular gas form in the very hot ICM? Revaz et al., (2008) performed numerical simulations to study the influence of the AGN on the formation of cold filaments. Along the radio-lobes (expanding in the ICM), where the hot gas is compressed, the over-dense regions can cool more efficiently. The simulations showed that buoyantly rising cavities in the ICM can drag out this cooling gas. When the gas is very cold, it can decouple from the outflowing gas and fall back onto the central galaxy from regions as far as $\sim 50 \mathrm{kpc}$, as observed in CO.

\section{What is the main source of excitation?}

Optical line ratios in BCGs are typical of Low Ionization Nuclear Emission Regions (LINER), (ie Heckman et al., 1989; Crawford et al., 1999). This is true even at large distance from the AGN (McDonald et al., 2011). Recently, Lim et al., (2012) mapped the $\mathrm{H}_{2}$ 1-0 in NGC 1275 and found $\mathrm{H}_{2} / \mathrm{H}_{\alpha}$ ratio of $\sim-0.08$ over the whole nebula. This ratio does not change with the distance from the AGN. So a direct influence of the AGN on the filaments excitation seems to be ruled out. The question of the main source of excitation is still open. The two mechanisms most widely studied are: (i) shocks (Ogle et al., 2010, Nesvadba et al., 2011, Guillard et al., 2012) and (ii) energetic particles (Ferland et al., 2008, 2009).

Molecular hydrogen Pure rotational $\mathrm{H}_{2} \mathrm{~S}(3) 9.67 \mu \mathrm{m}, \mathrm{S}(2) 12.28 \mu \mathrm{m}$, and $\mathrm{S}(1) 17.04 \mu \mathrm{m}$ lines were detected in BCGs by Spitzer (Egami et al., 2006). Those lines were then found in 9 other BCGs (Donahue et al., 2011): 2A0335, A478, A1068, A1795, A1835, A2597, HydraA, MS0735, PKS0745. Johnstone et al., (2007) also detected them in several filaments in NGC 1275. The $\mathrm{H}_{2}$ lines very are bright and the $\mathrm{H}_{2} / \mathrm{PAH}$ ratio is high $(\leqslant 0.03)$. So BCGs look like the MOHEGs (Molecular Hydrogen Emitting Galaxies) found by the Spitzer satellite. PDR models alone cannot explain such high line ratios (Guillard et al., 2012). Another source of energy is required to power the hydrogen emission lines. Cosmic Rays vs Kinetic energy dissipation Around radio-galaxies, Guillard et al., (2012) argues for AGN-winds energy dissipation by shocks. In BCG filaments, large scale kinetic energy dissipation is a possible mechanism that needs to be studied. The extra-energy required by the observations could also be provided by energetic particles (secondary electrons) collisional heating and ionization (Ferland et al., 2008, 2009). But this needs very high cosmic ray ionization rates. More recently, Fabian et al., (2011) proposed that this heating/ionization could be due to the hot surrounding gas penetrating the cold gas (hot/cold gas mixing) via magnetic reconnection.

\section{Cooling flows in the ALMA era}

The current mm-facilities have shown the existence of large amount of gas in BCGs. The emission is however very faint and few objects have been mapped. When resolved, the cold gas lies in extended filaments also visible in the optical and soft X-ray. Now is the time of ALMA, that will revolutionize our understanding of cooling flows.

More CO transitions, other molecules, dust The ALMA sensitivity and its submillimeter bands will open the access to high-J CO lines and new molecules, like observed in nearby star forming/radio-galaxies. ALMA will determine the dominant excitation mechanism in cooling flows and help understand the nature of the molecular gas around BCGs. It will also map in great details the dust pointed out by Herschel very recently.

Details of the central region The ALMA high spatial resolution and sensitivity will give access to the very central region, around the AGN. It will look for signs of accretion 
disks and molecular outflows and study the details of the AGN-fueling/feedback in BCGs.

Increase the sample, look for filaments ALMA will determine whether all BCGs are surrounded by large molecular filaments. It is a key step to understand the gas accretion mechanisms on galaxies and the possible influence of the AGN (that could enhanced local cooling and lead to the formation of cold/cooling filaments).

\section{References}

Allen S., Schmidt, R., \& et Fabian, A. C. 2001, MNRAS 328, 37

Bayet, E., Hartquist, T. W., Viti, S., Williams, D. A., \& Bell, T. A., 2010, A\&\&A, 521, 16

Bayet, E., Hartquist, T. W., Viti, S., Williams, D. A., \& Bell, T. A. 2011, MNRAS 414, 1583

Braine J. \& Dupraz C. 1994, A\& A 283, 407

Braine J., Wyrowski, F., Radford, S. J. E., Henkel, C., \& Lesch, H 1995, A\& A 293, 315

Bregman, Joel N., Miller, Eric D., Irwin, \& Jimmy A., 2001, ApJ 553, 125

Bridges T. J. \& Irwin J. A. 1998, MNRAS, 300, 967

Conselice, C. J., Gallagher, J. S., \& Wise, R. F. G. 2001, AJ, 122, 2281

Crawford C. S., Allen S. W., Ebeling H., Edge A. C, \& Fabian A. C. 1999 MNRAS, 306, 857

Donahue, Megan, de Messires, Genevive E., O'Connell, et al., 2011, ApJ, 732, 40

Edge A. C. 2001, MNRAS, 328, 762

Egami, E., Rieke, G. H., Fadda, D., \& Hines, D. C., 2006, ApJ, 652, 21

Fabian A. C. 1994, ARAA 32, 277

Fabian A. C. Sanders, J. S., Williams, R. J. R., Lazarian et al. 2011, MNRAS 418, 2154

Fabian A. C. 2012, ARAA 50, 455

Fabian, A. C., Sanders, J. S., Taylor, G. B. et al., 2006, MNRAS, 366, 417

Ferland, G. J., Fabian, A. C., Hatch, N. A., Johnstone, R. M. et al., \& MNRAS, 2008, 386, 72

Ferland G., Fabian, A. C. Hatch, N. A., et al. 2009, MNRAS, 392, 1475

Grabelski, D. \& Ulmer, M 1990, ApJ, 355, 401

Guillard, P., Ogle, P. M., Emonts, B. H. C., Appleton, et al., 2012, , ApJ, 747, 95

Hatch, N. A., Crawford, C. S., Fabian, A. C., \& Johnstone, R. M. J., 2005, MNRAS, 358, 765

Heckman, T. M., Baum, S. A., van Breugel, W. J. M., \& McCarthy, P., 1989, ApJ, 338, 48

Ho, I.-Ting, Lim, Jeremy, \& Dinh-V-Trung 2009, ApJ, 698, 1191

Inoue, M. Y., Kameno, S., Kawabe, R., Inoue, M., Hasegawa, T., \& Tanaka, M. 1996, AJ, 111

Johnstone, R. M., Hatch, N. A., Ferland, G. J., Fabian, A. C. et al., 2007, MNRAS, 382, 1246

Lazareff B., Castets A., Kim D. W., \& Jura M. 1989, ApJ 335, L13

Lim, J., Ao, Y., \& Trun, D-V. 2008, ApJ, 672, 252

Lim, Jeremy, Ohyama, Youichi, Chi-Hung, Yan, et al. 2012, ApJ, 744, 112

McDonald, Michael; Veilleux, Sylvain; \& Mushotzky, Richard, 2011, ApJ, 731, 33

McNamara B. R., \& Jaffe W. 1994, A\&A, 281, 673

Mirabel, I. F., Sanders, D. B., \& Kazes, I., 1989, ApJ, 340, 9

Nesvadba, N. P. H., Boulanger, F., Lehnert, M. D., Guillard, P., \& Salome, P., A\&্A, 2011, 536,5

O'Dea C. P., Baum S. A., Maloney P. M., et al., 1994, ApJ, 422, 467

Ogle, Patrick, Boulanger, Francois, Guillard, Pierre, et al., 2010, ApJ, 724, 1193

Peterson J. R., Paerels F. B. S., Kaastra J. S., Arnaud M., et al., 2001, A\&GA 365, L104

Reuter, H. P., Pohl, M., Lesch, H., \& Sievers, A. W. 1993, A\&SA, 277

Revaz Y., Combes F., \& Salomé P. 2008, A\&A, 477, 33

Salomé, P., \& Combes, F., 2003, A\& $A$, 412, 657

Salomé, P., Combes, F., Edge, A. C., et al. 2006, A\&BA, 454, 437

Salomé, P., Combes, F., Revaz, Y, et al. 2008a, A\&AA, 484, 317

Salomé, P., Revaz, Y., Combes, F., et al. 2008b, A\&A,483, 793

Salomé, P., Combes, F., Revaz, Y., al 2011, A\& $A$, 531, 85

Voigt, L. \& Fabian, A. C. 2004, MNRAS, 347, 1130

Wilman, R. J., Edge, A. C., \& Johnstone, R. M. 2005, MNRAS, 359, 75 